\title{
Pittosporum coccineum
}

\author{
Assessment by: Gemmill, C. et al.
}

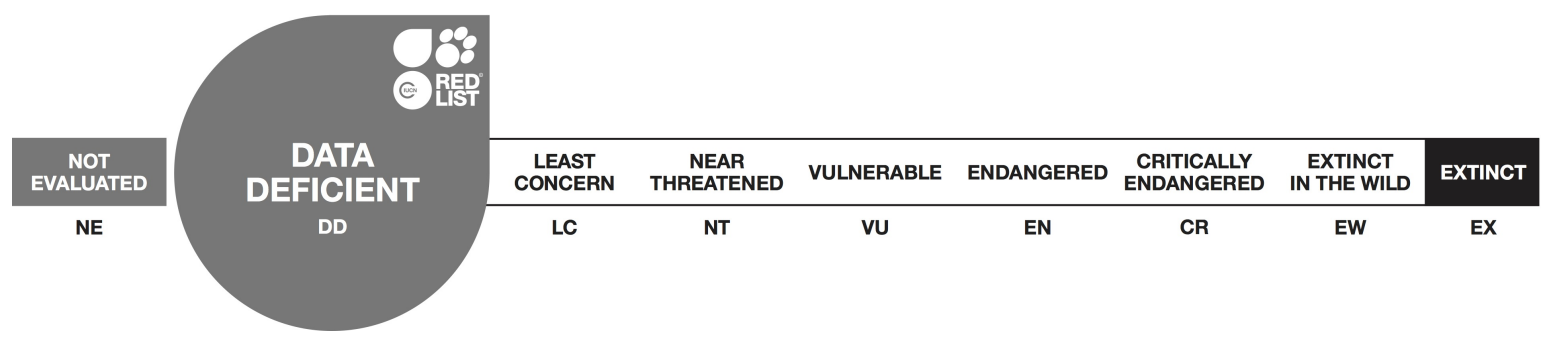

View on www.iucnredlist.org

Short citation: Gemmill, C. et al. 2017. Pittosporum coccineum. The IUCN Red List of Threatened Species 2017: e.T82947071A82951772. http://dx.doi.org/10.2305/IUCN.UK.20173.RLTS.T82947071A82951772.en [see full citation at end]

Copyright: (C 2017 International Union for Conservation of Nature and Natural Resources

Reproduction of this publication for educational or other non-commercial purposes is authorized without prior written permission from the copyright holder provided the source is fully acknowledged.

Reproduction of this publication for resale, reposting or other commercial purposes is prohibited without prior written permission from the copyright holder. For further details see Terms of Use.

The IUCN Red List of Threatened Species ${ }^{T M}$ is produced and managed by the IUCN Global Species Programme, the IUCN Species Survival Commission (SSC) and The IUCN Red List Partnership. The IUCN Red List Partners are: Arizona State University; BirdLife International; Botanic Gardens Conservation International; Conservation International; NatureServe; Royal Botanic Gardens, Kew; Sapienza University of Rome; Texas A\&M University; and Zoological Society of London.

If you see any errors or have any questions or suggestions on what is shown in this document, please provide us with feedback so that we can correct or extend the information provided. 


\section{Taxonomy}

\begin{tabular}{|c|c|c|c|c|}
\hline Kingdom & Phylum & Class & Order & Family \\
\hline Plantae & Tracheophyta & Magnoliopsida & Rosales & Pittosporaceae \\
\hline
\end{tabular}

Taxon Name: Pittosporum coccineum (Montr.) Beauvis.

\section{Synonym(s):}

- Quinsonia coccinea Montrouz.

\section{Taxonomic Source(s):}

Tirel, Ch. and Veillon, J.-M. 2002. Flore de la Nouvelle-Calédonie, tome 24. Pittosporaceae. Museum d'Histoire Naturelle, Paris.

\section{Assessment Information}

Red List Category \& Criteria: Data Deficient ver 3.1

Year Published: 2017

Date Assessed: July 24, 2015

\section{Justification:}

Pittosporum coccineum is an endemic small tree of New Caledonia, which, under the current delimitation of the taxon, is distributed on the northern Grande Terre, on both west and east coasts and in Belep. According to current phylogenetic studies, subpopulations located on the west coast, the east coast and Belep probably constitute three distinct taxonomic entities. P. coccineum is therefore classified as Data Deficient until the species delimitations are clarified.

\section{Geographic Range}

\section{Range Description:}

Pittosporum coccineum is an endemic small tree of New Caledonia present on the northern Grande Terre, on both west and east coasts and in Bélep.

\section{Country Occurrence:}

Native: New Caledonia 


\section{Distribution Map}

\section{Pittosporum coccineum}
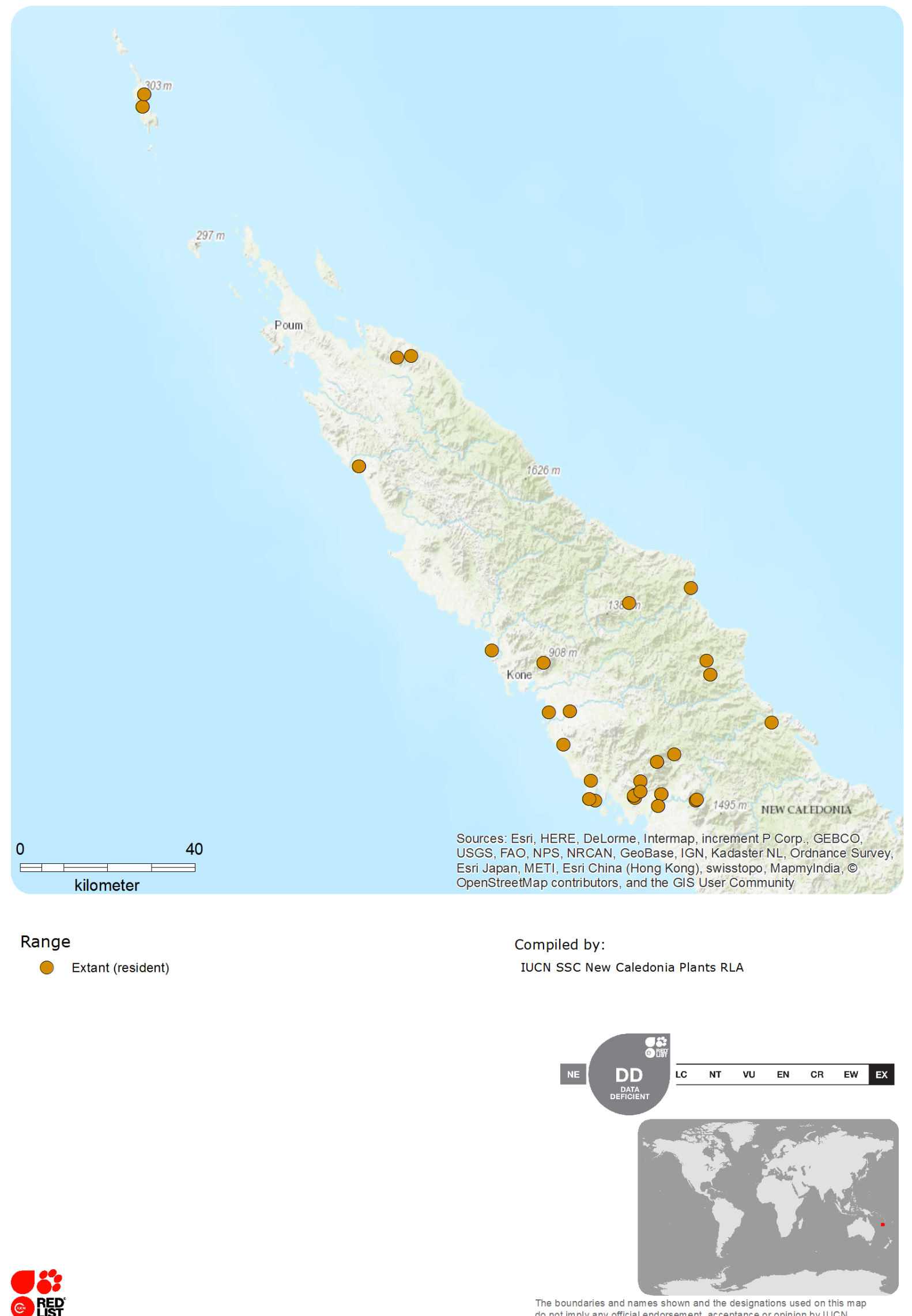


\section{Population}

Population size is unknown.

Current Population Trend: Unknown

\section{Habitat and Ecology (see Appendix for additional information)}

Pittosporum coccineum is found on all kind of substrates, in dense humid forest and in dry forest.

Systems: Terrestrial

\section{Threats (see Appendix for additional information)}

Due to its ecology and geographic range, Pittosporum coccineum could be affected by the majority of inventoried threats in New-Caledonia including fire, Rusa Deer, mining activities, animal husbandry and urbanization.

\section{Conservation Actions (see Appendix for additional information)}

Pittosporum coccineum is protected by legislation in Province Sud. This species occurs in one protected area of Province Nord: la réserve naturelle intégrale de la baie de Nékoro. In order to remove taxonomic doubts and thus properly assess the extinction risk of $P$. coccineum, it is urgent to finalize work on its phylogeny and to describe taxa which are included within the current species complex. According to Jean-Marie Veillon (pers. comm.), highlighted heterogeneity could be the result of inclusion of $P$. vieillardii under $P$. coccineum, which has the same floral morphology but differs in flower size.

\section{Credits}

Assessor(s): $\quad$ Gemmill, C., Veillon, J.-M., Amice, R., Cazé, H., Dumontet, V., Fleurot, D., Garnier, D., Letocart, I., Gâteblé, G., Letocart, D., Maggia, L. \& Pain, A.

Reviewer(s): $\quad$ Tanguy, V.

Facilitators(s) and Chanfreau, S.

Compiler(s): 


\section{Bibliography}

Endemia.nc. 2016. Faune et Flore de Nouvelle-Calédonie. Available at: http://www.endemia.nc.

Gomez, C., Mangeas, M., Curt, T., Ibanez, T., Munzinger, J., Dumas, P., Jérémy, A., Despinoy, M. and Hély, C. 2014. Wildfire risk for main vegetation units in a biodiversity hotspot: modeling approach in New Caledonia, South Pacific. Ecology and Evolution 5(2): 377-390. DOI: 10.1002/ece3.1317.

Hély-Alleaume. 2012. INC : Incendies et biodiversité des éco-systèmes en Nouvelle-Calédonie.

IUCN. 2017. The IUCN Red List of Threatened Species. Version 2017-3. Available at: www.iucnredlist.org. (Accessed: 7 December 2017).

L'Huillier, L., Jaffré, T. and Wulff, A. 2010. Mines et Environnement en Nouvelle-Calédonie: les milieux sur substrats ultramafiques et leur restauration. IAC, Nouméa.

Tirel, Ch. and Veillon, J.-M. 2002. Flore de la Nouvelle-Calédonie, tome 24. Pittosporaceae. Museum d'Histoire Naturelle, Paris.

\section{Citation}

Gemmill, C., Veillon, J.-M., Amice, R., Cazé, H., Dumontet, V., Fleurot, D., Garnier, D., Letocart, I., Gâteblé, G., Letocart, D., Maggia, L. \& Pain, A. 2017. Pittosporum coccineum. The IUCN Red List of Threatened Species 2017: e.T82947071A82951772. http://dx.doi.org/10.2305/IUCN.UK.20173.RLTS.T82947071A82951772.en

\section{Disclaimer}

To make use of this information, please check the Terms of Use.

\section{External Resources}

For Images and External Links to Additional Information, please see the Red List website. 


\section{Appendix}

\section{Habitats}

(http://www.iucnredlist.org/technical-documents/classification-schemes)

\begin{tabular}{|c|c|c|c|}
\hline Habitat & Season & Suitability & $\begin{array}{l}\text { Major } \\
\text { Importance? }\end{array}$ \\
\hline 1. Forest -> 1.5. Forest - Subtropical/Tropical Dry & - & Suitable & - \\
\hline 1. Forest -> 1.6. Forest - Subtropical/Tropical Moist Lowland & - & Suitable & - \\
\hline
\end{tabular}

\section{Threats}

(http://www.iucnredlist.org/technical-documents/classification-schemes)

\begin{tabular}{|c|c|c|c|c|}
\hline Threat & Timing & Scope & Severity & Impact Score \\
\hline \multirow{3}{*}{$\begin{array}{l}\text { 1. Residential \& commercial development }->1.1 . \\
\text { Housing \& urban areas }\end{array}$} & Ongoing & - & - & - \\
\hline & Stresses: & \multirow{2}{*}{\multicolumn{3}{|c|}{$\begin{array}{l}\text { 1. Ecosystem stresses }->1.1 \text {. Ecosystem conversion } \\
\text { 1. Ecosystem stresses }->1.2 \text {. Ecosystem degradation }\end{array}$}} \\
\hline & & & & \\
\hline \multirow{4}{*}{$\begin{array}{l}\text { 2. Agriculture \& aquaculture }->2.3 \text {. Livestock farming } \\
\& \text { ranching }->2.3 .2 \text {. Small-holder grazing, ranching or } \\
\text { farming }\end{array}$} & Ongoing & - & - & - \\
\hline & Stresses: & \multirow{3}{*}{\multicolumn{3}{|c|}{$\begin{array}{l}\text { 1. Ecosystem stresses }->1.1 \text {. Ecosystem conversion } \\
\text { 1. Ecosystem stresses }->1.2 \text {. Ecosystem degradation } \\
\text { 2. Species Stresses }->2.3 \text {. Indirect species effects }-> \\
\text { 2.3.7. Reduced reproductive success }\end{array}$}} \\
\hline & & & & \\
\hline & & & & \\
\hline \multirow{3}{*}{$\begin{array}{l}\text { 3. Energy production \& mining }->3.2 \text {. Mining \& } \\
\text { quarrying }\end{array}$} & Ongoing & - & - & - \\
\hline & Stresses: & \multirow{2}{*}{\multicolumn{3}{|c|}{$\begin{array}{l}\text { 1. Ecosystem stresses }->1.1 \text {. Ecosystem conversion } \\
\text { 1. Ecosystem stresses }->1.2 \text {. Ecosystem degradation }\end{array}$}} \\
\hline & & & & \\
\hline \multirow{4}{*}{$\begin{array}{l}\text { 7. Natural system modifications }->7.1 \text {. Fire } \& \text { fire } \\
\text { suppression }->7.1 .1 \text {. Increase in fire } \\
\text { frequency/intensity }\end{array}$} & Ongoing & - & - & - \\
\hline & Stresses: & \multirow{3}{*}{\multicolumn{3}{|c|}{$\begin{array}{l}\text { 1. Ecosystem stresses }->1.1 \text {. Ecosystem conversion } \\
\text { 1. Ecosystem stresses }->1.2 \text {. Ecosystem degradation } \\
\text { 2. Species Stresses }->\text { 2.1. Species mortality }\end{array}$}} \\
\hline & & & & \\
\hline & & & & \\
\hline \multirow{3}{*}{$\begin{array}{l}\text { 8. Invasive and other problematic species, genes } \& \\
\text { diseases }->8.1 \text {. Invasive non-native/alien } \\
\text { species/diseases }->\text { 8.1.2. Named species (Rusa } \\
\text { timorensis) }\end{array}$} & Ongoing & - & - & - \\
\hline & Stresses: & \multirow{2}{*}{\multicolumn{3}{|c|}{$\begin{array}{l}\text { 2. Species Stresses }->2.2 \text {. Species disturbance } \\
\text { 2. Species Stresses }->2.3 \text {. Indirect species effects }-> \\
\text { 2.3.7. Reduced reproductive success }\end{array}$}} \\
\hline & & & & \\
\hline
\end{tabular}

\section{Conservation Actions in Place}

(http://www.iucnredlist.org/technical-documents/classification-schemes) 


\begin{tabular}{|l|}
\hline Conservation Actions in Place \\
\hline In-Place Land/Water Protection and Management \\
\hline Occur in at least one PA: Yes \\
\hline Invasive species control or prevention: No \\
\hline In-Place Species Management \\
\hline Successfully reintroduced or introduced beningly: No \\
\hline Subject to ex-situ conservation: No \\
\hline
\end{tabular}

\title{
Research Needed
}

(http://www.iucnredlist.org/technical-documents/classification-schemes)

\section{Research Needed}

1. Research -> 1.1. Taxonomy

\section{Additional Data Fields}

\author{
Distribution \\ Estimated area of occupancy (AOO) $\left(\mathrm{km}^{2}\right): 124$ \\ Continuing decline in area of occupancy (AOO): Unknown \\ Extreme fluctuations in area of occupancy (AOO): No \\ Estimated extent of occurrence (EOO) $\left(\mathrm{km}^{2}\right): 8659$ \\ Continuing decline in extent of occurrence (EOO): Unknown \\ Extreme fluctuations in extent of occurrence (EOO): No \\ Continuing decline in number of locations: Unknown \\ Extreme fluctuations in the number of locations: No \\ Lower elevation limit (m): 1 \\ Upper elevation limit (m): 700 \\ Population \\ Continuing decline of mature individuals: Unknown \\ Extreme fluctuations: No \\ Population severely fragmented: No \\ Extreme fluctuations in subpopulations: No \\ Habitats and Ecology \\ Continuing decline in area, extent and/or quality of habitat: Unknown
}


Habitats and Ecology

Generation Length (years): 0 


\section{The IUCN Red List Partnership}

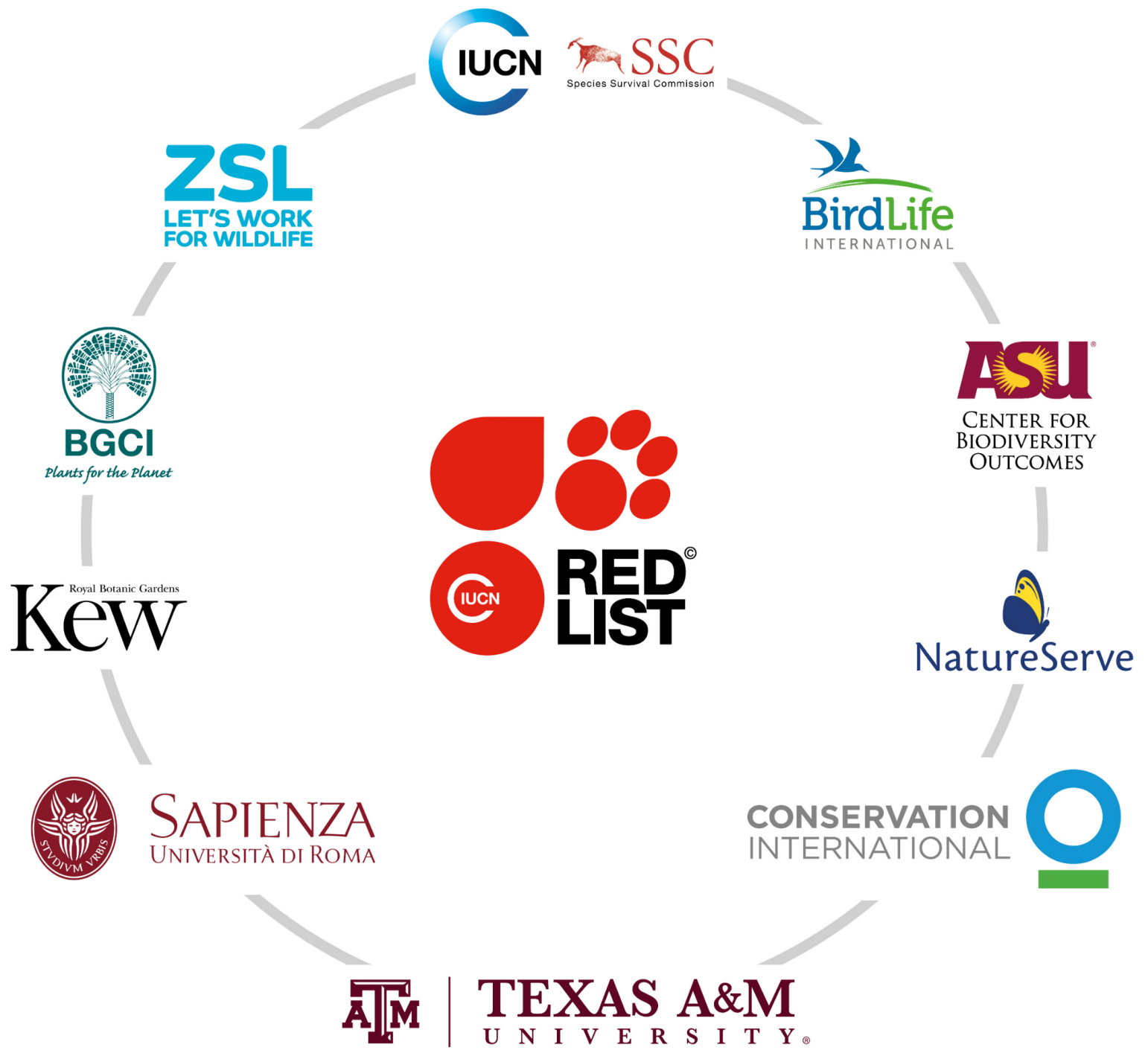

The IUCN Red List of Threatened Species ${ }^{\mathrm{TM}}$ is produced and managed by the IUCN Global Species Programme, the IUCN Species Survival Commission (SSC) and The IUCN Red List Partnership.

The IUCN Red List Partners are: Arizona State University; BirdLife International; Botanic Gardens Conservation International; Conservation International; NatureServe; Royal Botanic Gardens, Kew; Sapienza University of Rome; Texas A\&M University; and Zoological Society of London. 